\title{
Resolving blended radial velocities
}

\author{
Alexandre Santerne ${ }^{1,2}$, Claire Moutou ${ }^{1}$, François Bouchy ${ }^{2,3}$ and the \\ CoRoT Exoplanet Science Team \\ ${ }^{1}$ Laboratoire d'Astrophysique de Marseille, Université d'Aix-Marseille \& CNRS, \\ 38 rue Frédéric Joliot-Curie, 13388 Marseille cedex 13, France \\ email: alexandre.santerne@oamp.fr \\ ${ }^{2}$ Observatoire de Haute Provence, Université d'Aix-Marseille \& CNRS, \\ 04670 Saint Michel l'Observatoire, France \\ ${ }^{3}$ Institut d'Astrophysique de Paris, UMR7095 CNRS, Université Pierre \& Marie Curie, \\ 98bis boulevard Arago, 75014 Paris, France
}

\begin{abstract}
In space, photometric surveys are very efficient to detect small transiting planets or stars which are contaminated by blended eclipsing binaries. We present some simulations compared to radial velocity (RV) observations obtained with the SOPHIE spectrograph (OHP, France) in order to determine the true nature of a brown dwarf candidate revealed by CoRoT: a background eclipsing binary diluted by a foreground star.
\end{abstract}

Keywords. techniques: radial velocities, techniques: photometric, planetary systems

\section{Introduction}

CoRoT and Kepler space missions are detecting by high accuracy photometry a large number of transiting planetary candidates, with lots of neptune-like planets or SuperEarth candidates (e.g. CoRoT-7 (Léger et al. 2009) or Kepler-9d (Torres et al. 2011)). Radial velocity (RV) follow-up is required to discard eclipsing binaries from true planets and characterize the mass of the planet. In the regime of shallow transits, is expected a high frequency of diluted eclipsing binaries (Brown 2003) that could mimic both a transit and also the radial velocity signal of a planet. We perform simulations of precise radial velocity diagnostics to help identifying spectroscopic blends.

\section{A transiting brown dwarf candidate}

$\mathrm{A} \sim 0.3 \%$-depth transiting candidate with an orbital period of 2.25 days was detected in the CoRoT light curve. As shown in Fig. 1 (left pannel), the radial velocity follow-up made with the SOPHIE spectrograph (Bouchy et al. 2009) mounted on 1.93-m telescope in Observatoire de Haute Provence (France) indicate a clear RV signature compatible with a brown dwarf in phase with CoRoT ephemeris. But, bisector span also showed variations in anti-correlation with velocities (Fig. 1 middle pannel). FWHM variations are seen at both, the orbital period and half the orbital period (Fig. 1 right pannel).

\section{Blend simulations}

We performed blend simulations of this brown dwarf candidate in order to explain the observed RV diagnostics (RV, bisector and FWHM ). We simulated two blended CCF (Cross Correlation Function): the main one without any RV variation, the second one with variations in phase with the CoRoT ephemeris. The width and contrast of the primary $\mathrm{CCF}$ is fixed to the observed profile. We find, in this preliminary work, 

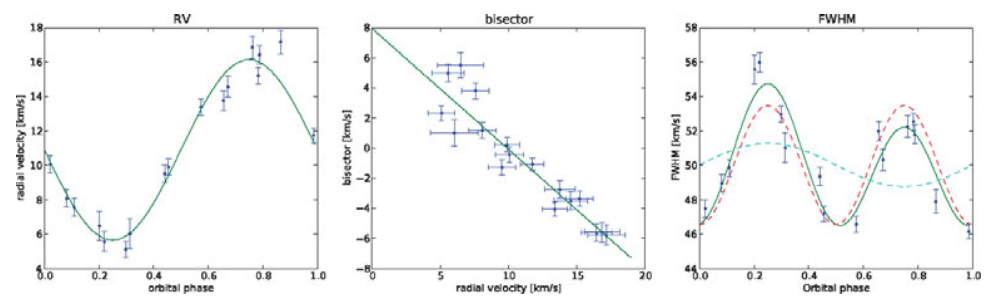

Figure 1. From left to right: phase-folded RV curve of this candidate with the best circular fit, bisector span against RV that show a clear anticorrelation and phase-folded FWHM curve that shows two variations, one at half the orbital period (dashed red line) and one at the orbital period (dashed-light blue line).
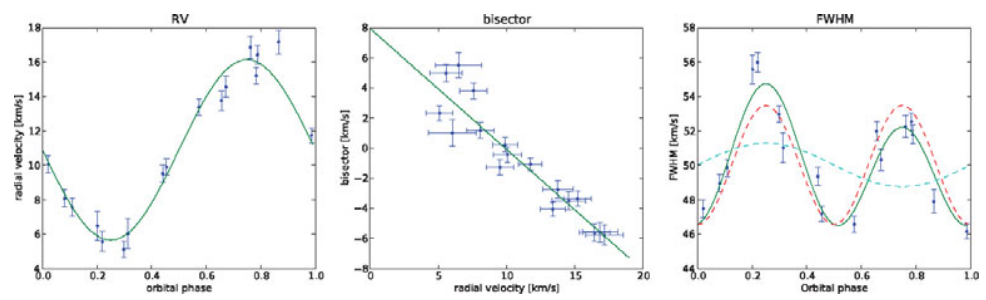

Figure 2. From left to right: simulated CCF of the main and secondary star, phase-folded simulated RV curve, simulated bisector against simulated RV, simulated FWHM as a function of the orbital phase.

that we could qualitatively reproduce RV data (see Fig.2) with a background spectroscopic and eclipsing binary which have the following characteristics: $\mathrm{K}_{\text {blend }} \sim 25 \mathrm{~km} . \mathrm{s}^{-1}$, $\Delta_{\text {flux }} \sim 14 \%, \mathrm{FWHM}_{\text {blend }} \sim 25 \mathrm{~km} . \mathrm{s}^{-1}, \mathrm{FWHM}_{\text {target }} \sim 50 \mathrm{~km} . \mathrm{s}^{-1}, \Delta_{v_{0}} \sim 100 \mathrm{~m} . \mathrm{s}^{-1}$.

The negative slope of the bisector seems to be caused by the fact that the secondary star is a lower-rotating star than the primary. FWHM variations at half the orbital period are caused by the secondary star which increases the measured FWHM of the primary at extremal phases, when variations at the orbital period are caused by the slight RV offset between the main star and the binary.

\section{Conclusion}

Futur space-based surveys, like PLATO or TESS, will find amount of small-transit candidates where part of them will be blended by background stars. This kind of simulations, based on RV CCF diagnostics will be very useful to characterize the observed systems or to discard background blends (Santerne et al. in prep). The behaviour of the bisector span with respect to the RV appears as a very sensitive diagnostics of distant spectroscopic binaries. This tools of RV simulations will be completed soon by transit simulations as in Torres et al. (2011) and by a robust statistical analysis of best solutions.

\section{References}

Bouchy, F., Hébrard, G., Udry, S., et al. 2009, A\&A, 505, 853

Brown, T. M. 2003, ApJ (Letters), 593, L125

Léger, A., Rouan, D., Schneider, J., et al. 2009, A\&A, 506, 287

Torres, G., Fressin, F., Batalha, N. M., et al. 2011, ApJ, 727, id.24 